\title{
Low Albumin Levels, More Than Morbid Obesity, Are Associated With Complications After TKA
}

\author{
Charles L. Nelson MD, Nabil M. Elkassabany MD, MSCE, \\ Atul F. Kamath MD, Jiabin Liu MD, PhD
}

Received: 18 January 2015/ Accepted: 22 April 2015/Published online: 21 May 2015

(C) The Association of Bone and Joint Surgeons \& 2015

\begin{abstract}
Background Morbid obesity and malnutrition are thought to be associated with more frequent perioperative complications after TKA. However, morbid obesity and malnutrition often are co-occurring conditions. Therefore it is important to understand whether morbid obesity, malnutrition, or both are independently associated with more frequent perioperative complications. In addition, assessing the magnitude of an increase in complications and whether these complications are major or minor is important for both conditions.

Questions/purposes We asked: (1) Is morbid obesity independently associated with more frequent major perioperative complications after TKA? (2) Are major
\end{abstract}

One of the authors certifies that he (CLN), or a member of his or her immediate family, has or may receive payments or benefits, during the study period, an amount of USD 10,000-USD 100,000 from Zimmer Inc (Warsaw, IN, USA).

All ICMJE Conflict of Interest Forms for authors and Clinical Orthopaedics and Related Research ${ }^{\mathbb{R}}$ editors and board members are on file with the publication and can be viewed on request.

Each author certifies that his or her institution approved or waived approval for the human protocol for this investigation and that all investigations were conducted in conformity with ethical principles of research.

This work performed at the University of Pennsylvania, Philadelphia, PA, USA.

C. L. Nelson ( $\square)$, A. F. Kamath

Department of Orthopaedic Surgery, Perelman School of

Medicine, University of Pennsylvania, PMUC, 3737 Market

Street, Suite 600, Philadelphia, PA 19104, USA

e-mail: charles.nelson@uphs.upenn.edu;

clenwoodnelson@gmail.com

N. M. Elkassabany, J. Liu

Department of Anesthesiology \& Critical Care, Perelman School of Medicine, University of Pennsylvania, Philadelphia, PA, USA perioperative complications after TKA more prevalent among patients with a low serum albumin?

Methods The National Surgical Quality Improvement Program (NSQIP) database was analyzed from 2006 to 2013. Patients were grouped as morbidly obese (BMI $\geq 40$ $\mathrm{kg} / \mathrm{m}^{2}$ ) or nonmorbidly obese (BMI $\geq 18.5 \mathrm{~kg} / \mathrm{m}^{2}$ to $<40$ $\mathrm{kg} / \mathrm{m}^{2}$ ), or by low serum albumin (serum albumin level $<3.5 \mathrm{mg} / \mathrm{dL}$ ) or normal serum albumin (serum albumin level $\geq 3.5 \mathrm{mg} / \mathrm{dL}$ ). The study cohort included 77,785 patients, including 35,573 patients with a serum albumin level of $3.5 \mathrm{~g} / \mathrm{dL}$ or greater and 1570 patients with a serum albumin level less than $3.5 \mathrm{~g} / \mathrm{dL}$. Therefore, serum albumin levels were available for only 37,173 of the 77,785 of the patients $(48 \%)$. There were 66,382 patients with a BMI between $18.5 \mathrm{~kg} / \mathrm{m}^{2}$ and $40 \mathrm{~kg} / \mathrm{m}^{2}$ and 11,403 patients with a BMI greater than $40 \mathrm{~kg} / \mathrm{m}^{2}$. Data were recorded on patient mortality along with 21 complications reported in the NSQIP. We also developed three composite complication variables to represent risk of any infections, cardiac or pulmonary complications, and any major complications. For each complication, multivariate logistic regression analysis was performed. Independent variables included patient age, sex, race, BMI, American Society of Anesthesiologists classification, year of surgery, and Charlson comorbidity index score.

Results Mortality was not increased in the morbidly obese group $(0.14 \%$ vs $0.14 \% ; \mathrm{p}=0.942)$. Patients who were morbidly obese were more likely to have progressive renal insufficiency $(0.30 \%$ vs $0.10 \%$; odds ratio [OR], 2.47; 95\% CI, 1.27-4.29; $\mathrm{p}<0.001)$, superficial infection (1.07\% vs $0.55 \%$; OR, 1.87 ; 95\% CI, $1.39-2.51$; p < $0.001)$, and sepsis $(0.36 \%$ vs $0.23 \%$; OR, $1.70 ; 95 \% \mathrm{CI}$, $1.04-2.53 ; \mathrm{p}=0.034)$ compared with patients who were not morbidly obese. Patients who were morbidly obese were less likely to require blood transfusion $(8.68 \%$ vs 
12.06\%; OR, 0.70; 95\% CI, 0.63-0.77; p < 0.001) compared with patients who were not morbidly obese. Morbid obesity was not associated with any of the other 21 perioperative complications recorded in the NSQIP database. With respect to the composite complication variables, patients who were morbidly obese had an increased risk of any infection $(3.31 \%$ vs $2.41 \%$; OR, 1.38 ; $95 \%$ CI, 1.16 $1.64 ; \mathrm{p}<0.001$ ) but not for cardiopulmonary or any major complication. The group with low serum albumin had higher mortality than the group with normal serum albumin $(0.64 \%$ vs $0.15 \%$; OR, $3.17 ; \quad 95 \%$ CI, 1.58-6.35; $\mathrm{p}=0.001$ ). Patients in the low serum albumin group were more likely to have a superficial surgical site infection $(1.27 \%$ vs $0.64 \%$; OR, 1.27 ; 95\% CI, 1.09-2.75; $\mathrm{p}=0.020)$; deep surgical site infection $(0.38 \%$ vs $0.12 \%$; OR, 3.64; 95\% CI, 1.54-8.63; $\mathrm{p}=0.003)$; organ space surgical site infection $(0.45 \%$ vs $0.15 \%$; OR, $2.71 ; 95 \% \mathrm{CI}$, $1.23-5.97 ; \mathrm{p}=0.013)$; pneumonia (1.21 vs $0.29 \%$; OR, 3.55; 95\% CI, 2.14-5.89; $\mathrm{p}<0.001)$; require unplanned intubation $(0.51 \%$ vs $0.17 \%$, OR, 2.24; $95 \%$ CI, $1.07-4.69$; $\mathrm{p}=0.033$ ); and remain on a ventilator more than 48 hours $(0.38 \%$ vs $0.07 \%$; OR, $4.03 ; \quad 95 \%$ CI, 1.64-9.90; $\mathrm{p}=0.002)$. They are more likely to have progressive renal insufficiency $(0.45 \%$ vs $0.12 \%$; OR, $2.71 ; 95 \%$ CI, $1.21-$ $6.07 ; \mathrm{p}=0.015)$; acute renal failure $(0.32 \%$ vs $0.06 \%$; $\mathrm{OR}$, 5.19 ; 95\% CI, 1.96-13.73; $\mathrm{p}=0.001)$; cardiac arrest requiring cardiopulmonary resuscitation $(0.19 \%$ vs $0.12 \%$; OR, 3.74; 95\% CI, 1.50-9.28; $\mathrm{p}=0.005)$; and septic shock $(0.38 \%$ vs $0.08 \%$; OR, $4.4 ; \quad 95 \%$ CI, 1.74-11.09; $\mathrm{p}=0.002$ ). Patients in the low serum albumin group also were more likely to require blood transfusion $(17.8 \%$ vs 12.4\%; OR, 1.56; 95\% CI, 1.35-1.81; $\mathrm{p}<0.001$ ). In addition, among the three composite complication variables, any infection (5.0\% vs 2.4\%; OR, 2.0; 95\% CI, 1.53-2.61; p < $0.001)$ and any major complication (2.4\% vs $1.3 \%$; OR, $1.41 ; 95 \% \mathrm{CI}, 1.00-1.97 ; \mathrm{p}=0.050$ ) were more prevalent among the patients with low serum albumin. There was no difference for cardiopulmonary complications.

Conclusions Morbid obesity is not independently associated with the majority of perioperative complications measured by the NSQIP and was associated only with increases in progressive renal insufficiency, superficial surgical site infection, and sepsis among the 21 perioperative variables measured. However, low serum albumin was associated with increased mortality and multiple additional major perioperative complications after TKA. Low serum albumin, more so than morbid obesity, is associated with major perioperative complications. This is an important finding, as low serum albumin may be more modifiable than morbid obesity in patients who are immobile or have advanced knee osteoarthritis.

Level of Evidence Level III, prognostic study.

\section{Introduction}

TKA is a highly successful and cost-effective treatment for patients with advanced osteoarthritis (OA) of the knee [17]. Benefits of TKA in populations at greater risk for complications are less well defined. Morbid obesity and malnutrition have been shown to be associated with increased perioperative complications after TKA $[5,6,10$, $12,16,18,19,27,32]$; however, some studies have not shown an increased risk of perioperative complications in patients who are morbidly obese [3, 13, 14, 23, 25]. In addition, patients with morbid obesity are more likely to be malnourished than patients who are not obese, raising the question whether morbid obesity, malnutrition, or both, are independently associated with increased perioperative complications [12]. A previous study of 670 patients undergoing primary THA or TKA at our institution showed that low serum albumin, but not morbid obesity, was independently associated with a greater risk of perioperative complications.

Morbid obesity and malnutrition are considered modifiable risk factors by some surgeons. Although modest weight loss has been successful after intensive, supervised lifestyle modification or bariatric surgery in patients with sufficient mobility to exercise, sustained weight loss has not been achieved reliably [30]. It is not clear whether morbid obesity is truly modifiable, particularly in patients with limited mobility, difficulty exercising, and advanced knee OA. Some surgeons consider morbid obesity to be a relative contraindication to elective TKA [1].

Morbid obesity and advanced knee OA with disability warranting TKA often co-occur in patients. Restricting patients with morbid obesity from undergoing TKA would prevent many patients who are severely disabled from having access to a highly successful and cost-effective intervention. Furthermore, although some studies have shown inferior functional outcomes in patients with morbid obesity, numerous investigators have reported that these patients have equal or better satisfaction and functional improvement after TKA, with acceptable long-term outcomes $[3,8,14,20,26,28]$, compared with patients not so affected.

Our study was conducted to evaluate the roles of morbid obesity and low serum albumin in a large representative cohort with respect to perioperative complications within the first 30 days postoperatively.

\section{Methods}

Our study was exempted by our institutional review board. We retrospectively analyzed the American College of 


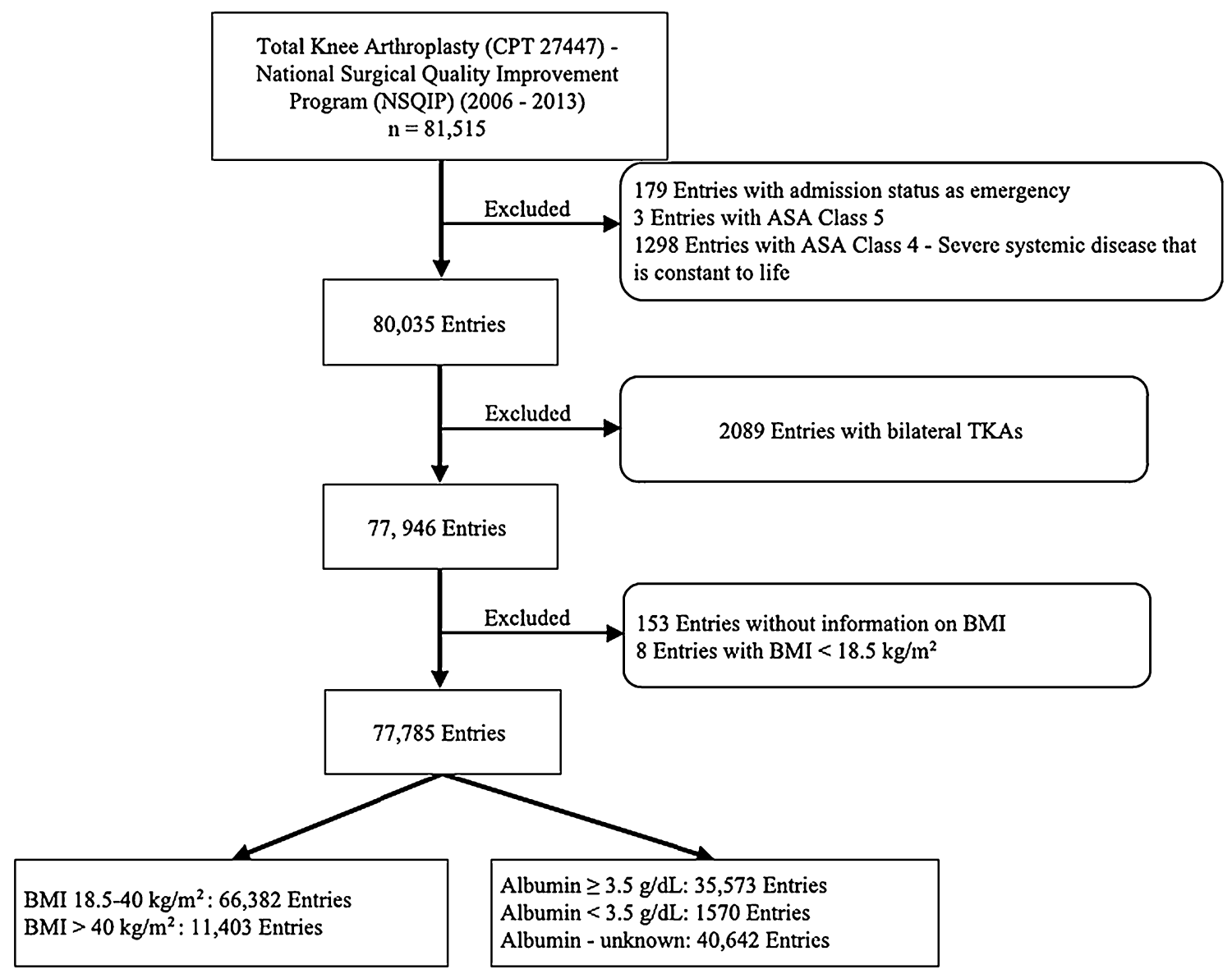

Fig. 1 A flowchart shows the patient selection process. CPT = current procedural terminology; ASA = American Society of Anesthesiologists.

Surgeons National Surgical Quality Improvement Program (NSQIP) database [2] from 2006 to 2013.

Our study cohort included patients with the Current Procedural Terminology (CPT) code for primary TKA (code 27447 ) as the principal procedure. There were a total of 81,515 identified entries in the NSQIP. Exclusion criteria were American Society of Anesthesiologists (ASA) Class 4 (1298 entries) and Class 5 (three entries); "Emergency" admission status (179 entries); BMI $<18.5 \mathrm{~kg} / \mathrm{m}^{2}$ (eight entries); and patients who had bilateral TKAs defined by a relevant concurrent CPT (2089 entries). Furthermore, patients with missing BMI data (153 entries) were excluded. The final study cohort included 77,785 patients (Fig. 1).

Patients were grouped as morbidly obese (BMI $\geq 40 \mathrm{~kg}$ / $\mathrm{m}^{2}$ ) or nonmorbidly obese (BMI between 18.5 and $40 \mathrm{~kg} /$ $\mathrm{m}^{2}$ ); and as having a low serum albumin level (serum albumin level $<3.5 \mathrm{mg} / \mathrm{dL}$ ) or normal serum albumin level (serum albumin level $\geq 3.5 \mathrm{mg} / \mathrm{dL}$ ). In addition to mortality, we analyzed data on 21 complications reported in the NSQIP database: superficial wound infection, deep incisional wound infection, organ space surgical site infection, surgical wound disruption, pneumonia, unplanned intubation, pulmonary embolism, on a ventilator more than 48 hours, progressive renal insufficiency, acute renal failure, urinary tract infection, stroke or cerebrovascular accident, coma more than 24 hours, peripheral nerve injury, cardiac arrest requiring cardiopulmonary resuscitation, myocardial infarction, blood transfusion, prosthesis failure, deep vein thrombosis, sepsis, and septic shock. Three composite complication variables were developed to represent risk of any infections (including superficial wound infection, deep incisional wound infection, organ space surgical site infection, surgical wound disruption, pneumonia, urinary tract infection, sepsis, and septic shock); cardiac or pulmonary complications (including unplanned intubation, pulmonary embolism, on ventilator more than 48 hours, cardiac arrest requiring cardiopulmonary resuscitation, and myocardial infarction); and any major complications (including unplanned intubation, pulmonary embolism, on ventilator more than 48 hours, progressive renal insufficiency, acute renal failure, stroke/cerebrovascular accident, coma more than 24 hours, cardiac arrest requiring cardiopulmonary resuscitation, myocardial infarction, sepsis, and septic shock).

Patient demographic information was analyzed (Table 1). There were several differences in the prevalence of 
Table 1. Demographic information of the patients $(n=77,785)$

\begin{tabular}{|c|c|c|c|c|c|c|}
\hline \multirow[t]{2}{*}{ Variable } & \multirow{2}{*}{$\begin{array}{l}\text { BMI: } 18.5-40 \mathrm{~kg} / \mathrm{m}^{2} \\
\text { Number }(\%)\end{array}$} & \multicolumn{2}{|c|}{$\mathrm{BMI}>40 \mathrm{~kg} / \mathrm{m}^{2}$} & \multicolumn{3}{|c|}{ Albumin $\geq 3.5 \mathrm{~g} / \mathrm{dL}$} \\
\hline & & Number $(\%)$ & $\mathrm{p}$ value & Number $(\%)$ & $\begin{array}{l}\text { Albumin }<3.5 \mathrm{~g} / \mathrm{dL} \\
\text { Number }(\%)\end{array}$ & $\mathrm{p}$ value \\
\hline Total subjects (number) & 66,382 & 11,403 & & 35,573 & 1570 & \\
\hline Age (years) & $67.9 \pm 9.9$ & $62.1 \pm 8.5$ & $<0.001$ & $67.0 \pm 9.9$ & $68.4 \pm 11.1$ & $<0.001$ \\
\hline \multicolumn{7}{|l|}{ Sex } \\
\hline Male & $25,700(38.8)$ & $2890(25.4)$ & $<0.001$ & $13,052(36.8)$ & $477(30.4)$ & $<0.001$ \\
\hline Female & $40,569(61.2$ & 8501 (74.6) & & $22,429((63.2)$ & $1090(69.9)$ & \\
\hline \multicolumn{7}{|l|}{ Race } \\
\hline White & $50,639(84.6)$ & 8618 (81.9) & $<0.001$ & $27,824(83.7)$ & $1199(79.9)$ & $<0.001$ \\
\hline Black & $3773(6.3)$ & $1247(7.1)$ & & $2309(6.9)$ & $169(11.3)$ & \\
\hline Hispanic & $3468(5.8)$ & $498(5.6)$ & & $2191(6.6)$ & 99 (6.6) & \\
\hline Others & 2008 (3.4) & $155(3.1)$ & & $926(2.8)$ & $33(2.2)$ & \\
\hline
\end{tabular}

preexisting comorbidities among study groups (Table 2). Patients with morbid obesity had higher ASA classification, Charlson comorbidity index score, and rates of dyspnea, hypertension, chronic obstructive pulmonary disease, and diabetes. The patients who were not morbidly obese had higher prevalences of coronary artery disease, peripheral vascular disease, and central nervous system disease (Table 2). Similar trends also existed between patients with low serum albumin and those with normal serum albumin.

All data analyses were performed using STATA ${ }^{\circledR} 12.1$ statistical software (StataCorp LP, College Station, TX, USA). Continuous variables were analyzed via t-test, and categorical variables were analyzed with Fisher's exact test and chi-square test. For each complication, multivariate logistic regression analysis was used to evaluate association with obesity and hypoalbuminemia, while compensating for potential confounding by preexisting comorbidities and other patient factors. The process used backward elimination with a threshold of $\mathrm{p}$ less than 0.2 for variable inclusion. The independent variables included patient age, sex, race, BMI, ASA classification, year of surgery, and Charlson comorbidity index score. Adjusted odds ratio (OR), 95\% CIs, and $\mathrm{p}$ values were reported. Each regression model was evaluated with the $\mathrm{C}$ statistic for model adequacy.

\section{Results}

\section{Morbid Obesity and Postoperative Complications}

Mortality was not increased in the morbidly obese group $(0.14 \%$ vs $0.14 \% ; p=0.942)$ (Table 3$)$. Patients with morbid obesity were more likely to have progressive renal insufficiency $(0.30 \%$ vs $0.10 \%$; OR, $2.47 ; 95 \%$ CI,
1.27-4.29; $\mathrm{p}<0.001$, superficial infection $(1.07 \%$ vs $0.55 \%$; OR, 1.87; 95\% CI, 1.39-2.51; p < 0.001), and sepsis $(0.36 \%$ vs $0.23 \%$; OR, $1.70 ; 95 \%$ CI, 1.04-2.53; $\mathrm{p}=0.034)$ compared with patients not so affected. Patients with morbid obesity were less likely to require a blood transfusion $(8.68 \%$ vs $12.06 \%$; p < 0.001; OR, 0.70; 95\% CI, 0.63-0.77; p < 0.001) compared with patients without morbid obesity. Morbid obesity was not associated with any of the other 21 perioperative complications recorded in the NSQIP database (Fig. 2). Among the three composite variables, morbid obesity was not associated with cardiac pulmonary complications $(1.03 \%$ vs $1.07 \%$; $\mathrm{p}=0.706)$ or any major complication $(1.56 \%$ vs $1.44 \%$; $\mathrm{p}=0.320)$, but was associated with an increased risk of any infection (3.3 vs 2.4; OR, 1.38; 95\% CI, 1.16-1.64; p < 0.001 ), owing predominantly to the increased rate of superficial surgical site infection (Table 4).

Low Serum Albumin and Postoperative Complications

The incidence of perioperative mortality was low in the nonmorbidly obese and morbidly obese groups $(0.14 \%$ vs $0.14 \% ; \mathrm{p}=0.942$ ) (Table 3 ), but significantly higher in the low serum albumin group compared with the group with normal serum albumin $(0.64 \%$ vs $0.15 \%$; OR, 3.17 ; 95\% CI, 1.58-6.35; $\mathrm{p}=0.001)$. Patients in the low serum albumin group were more likely to have superficial surgical site infection $(1.27 \%$ vs $0.64 \%$; OR, 1.27 ; 95\% CI, 1.09 $2.75 ; \mathrm{p}=0.020)$; deep surgical site infection $(0.38 \%$ vs $0.12 \%$; OR, 3.64; 95\% CI, 1.54-8.63; $\mathrm{p}=0.003)$; organ space surgical site infection $(0.45 \%$ vs $0.15 \%$; OR, 2.71 ; 95\% CI, 1.23-5.97; $\mathrm{p}=0.013)$; pneumonia (1.21 vs $0.29 \%$; OR, 3.55; 95\% CI, 2.14-5.89; $\mathrm{p}<0.001)$; require unplanned intubation $(0.51 \%$ vs $0.17 \%$, OR, $2.24 ; 95 \% \mathrm{CI}$, $1.07-4.69 ; \mathrm{p}=0.033)$; remain on a ventilator more than 48 
Table 2. Prevalence of comorbidities among patients $(n=77,785)$

\begin{tabular}{|c|c|c|c|c|c|c|c|c|c|c|}
\hline \multirow[t]{2}{*}{ Comorbidity variable } & \multicolumn{2}{|c|}{ BMI $18.5-40 \mathrm{~kg} / \mathrm{m}^{2}$} & \multicolumn{2}{|c|}{$\mathrm{BMI}>40 \mathrm{~kg} / \mathrm{m}^{2}$} & \multirow[t]{2}{*}{$\mathrm{p}$ value } & \multicolumn{2}{|c|}{ Albumin $\geq 3.5 \mathrm{~g} / \mathrm{dL}$} & \multicolumn{2}{|c|}{ Albumin $<3.5 \mathrm{~g} / \mathrm{dL}$} & \multirow[t]{2}{*}{$\mathrm{p}$ value } \\
\hline & Number & $\%$ & Number & $\%$ & & Number & $\%$ & Number & $\%$ & \\
\hline Total Subjects (number) & 66,309 & & 11,389 & & & 35,526 & & 1566 & & \\
\hline \multicolumn{11}{|l|}{ ASA classification } \\
\hline 1 & 1593 & 2.40 & 42 & 0.37 & $<0.001$ & 597 & 1.68 & 9 & 0.57 & $<0.001$ \\
\hline 2 & 36,999 & 55.80 & 3049 & 26.77 & & 17,967 & 50.57 & 500 & 31.93 & \\
\hline 3 & 27,717 & 41.80 & 8298 & 72.86 & & 16,962 & 47.75 & 1057 & 67.50 & \\
\hline \multicolumn{11}{|l|}{ Charlson comorbidity index } \\
\hline 0 & 53,143 & 80.06 & 7762 & 68.07 & $<0.001$ & 27,501 & 77.31 & 1074 & 68.41 & $<0.001$ \\
\hline 1 & 2490 & 3.75 & 385 & 3.38 & & 1304 & 3.67 & 115 & 7.32 & \\
\hline 2 & 9835 & 14.82 & 2964 & 25.99 & & 6193 & 17.41 & 317 & 20.19 & \\
\hline$\geq 3$ & 914 & 1.38 & 292 & 2.56 & & 575 & 1.62 & 64 & 4.08 & \\
\hline \multicolumn{11}{|l|}{ Functional health status before surgery } \\
\hline Dependent & 48 & 0.07 & 9 & 0.08 & 0.044 & 27 & 0.08 & 3 & 0.19 & $<0.001$ \\
\hline Partially dependent & 1260 & 1.91 & 256 & 2.26 & & 680 & 1.92 & 79 & 5.04 & \\
\hline $\begin{array}{l}\text { Congestive heart failure in } 30 \text { days } \\
\text { before surgery }\end{array}$ & 116 & 0.17 & 36 & 0.32 & 0.002 & 69 & 0.19 & 9 & 0.57 & 0.001 \\
\hline Coronary artery disease & 2164 & 10.40 & 258 & 7.16 & $<0.001$ & 1050 & 10.60 & 55 & 9.89 & 0.598 \\
\hline Peripheral vascular disease & 130 & 0.62 & 12 & 0.33 & 0.033 & 62 & 0.63 & 3 & 0.54 & 0.801 \\
\hline Hypertension requiring medication & 43,073 & 64.89 & 8761 & 76.83 & $<0.001$ & 24,203 & 68.04 & 1161 & 73.95 & $<0.001$ \\
\hline \multicolumn{11}{|l|}{ Dyspnea } \\
\hline At rest & 128 & 0.19 & 38 & 0.33 & $<0.001$ & 81 & 0.23 & 6 & 0.38 & $<0.001$ \\
\hline Moderate exertion & 3905 & 5.88 & 1413 & 12.39 & & 2593 & 7.29 & 205 & 13.06 & \\
\hline History of severe COPD & 2133 & 3.21 & 504 & 4.42 & $<0.001$ & 1291 & 3.63 & 120 & 7.64 & $<0.001$ \\
\hline End stage liver disease & 14 & 0.02 & 2 & 0.02 & 0.807 & 6 & 0.02 & 4 & 0.25 & $<0.001$ \\
\hline Renal failure & 84 & 0.13 & 14 & 0.12 & 0.917 & 46 & 0.13 & 13 & 0.83 & $<0.001$ \\
\hline \multicolumn{11}{|l|}{ Diabetes mellitus } \\
\hline Insulin & 2285 & 3.44 & 916 & 8.03 & $<0.001$ & 1513 & 4.25 & 137 & 8.73 & $<0.001$ \\
\hline Oral/noninsulin & 8080 & 12.17 & 2302 & 20.19 & & 5043 & 14.18 & 223 & 14.20 & \\
\hline Central nervous system disease & 1165 & 5.60 & 151 & 4.19 & 0.001 & 515 & 5.20 & 47 & 8.45 & 0.001 \\
\hline Spinal cord injury & 69 & 0.33 & 7 & 0.19 & 0.171 & 47 & 0.47 & 1 & 0.18 & 0.317 \\
\hline $\begin{array}{l}\text { Disseminated cancer, chemo-therapy / } \\
\text { radiotherapy }\end{array}$ & 122 & 0.18 & 8 & 0.07 & 0.006 & 74 & 0.21 & 7 & 0.45 & 0.048 \\
\hline Bleeding disorders & 1758 & 2.65 & 296 & 2.60 & 0.747 & 902 & 2.54 & 101 & 6.43 & $<0.001$ \\
\hline Prior operation within 30 days & 62 & 0.30 & 8 & 0.22 & 0.436 & 31 & 0.31 & 6 & 1.06 & 0.003 \\
\hline
\end{tabular}

$\mathrm{ASA}=$ American Society of Anesthesiologists; COPD = chronic obstructive pulmonary disease.

hours $(0.38 \%$ vs $0.07 \%$; OR, 4.03 ; $95 \%$ CI, $1.64-9.90$; $\mathrm{p}=0.002)$; and have progressive renal insufficiency $(0.45$ $\%$ vs $0.12 \%$; OR, $2.71 ; 95 \%$ CI, $1.21-6.07 ; \mathrm{p}=0.015)$; acute renal failure $(0.32 \%$ vs $0.06 \%$; OR, $5.19 ; 95 \% \mathrm{CI}$, $1.96-13.73 ; \mathrm{p}=0.001)$; cardiac arrest requiring cardiopulmonary resuscitation $(0.19 \%$ vs $0.12 \%$; OR, 3.74; 95\% CI, $1.50-9.28 ; \mathrm{p}=0.005)$; and septic shock $(0.38 \%$ vs $0.08 \%$; OR, 4.4; 95\% CI, 1.74-11.09; $\mathrm{p}=0.002$ ). Patients in the low serum albumin group also were more likely to require blood transfusion $(17.8 \%$ vs $12.4 \%$; OR, 1.56; 95\% CI, 1.35-1.81; $\mathrm{p}<0.001)$. In addition, among the three composite complication variables, any infection
(5.0\% vs $2.4 \%$; OR, 2.0; 95\% CI, 1.53-2.61; p < 0.001) and any major complication (2.4\% vs $1.3 \%$; OR, $1.41 ; 95 \%$ CI, 1.00-1.97; $\mathrm{p}=0.050$ ) were more prevalent among the patients with low serum albumin. We saw no differences in rates of cardiopulmonary complications.

\section{Discussion}

Morbid obesity and malnutrition are thought to be associated with more frequent perioperative complications in patients after TKA [5, 6, 10, 12, 31]. In addition, both often 
Table 3. Incidences of perioperative complications $(n=77,785)$

\begin{tabular}{|c|c|c|c|c|c|c|c|c|c|c|}
\hline \multirow[t]{2}{*}{ Complication } & \multicolumn{2}{|c|}{ BMI $18.5-40 \mathrm{~kg} / \mathrm{m}^{2}$} & \multicolumn{2}{|c|}{$\mathrm{BMI} \geq 40 \mathrm{~kg} / \mathrm{m}^{2}$} & \multirow[t]{2}{*}{$\mathrm{p}$ value } & \multicolumn{2}{|c|}{ Albumin $\geq 3.5 \mathrm{~g} / \mathrm{dL}$} & \multicolumn{2}{|c|}{ Albumin $<3.5 \mathrm{~g} / \mathrm{dL}$} & \multirow[t]{2}{*}{$\mathrm{p}$ value } \\
\hline & Number & $\%$ & Number & $\%$ & & Number & $\%$ & Number & $\%$ & \\
\hline $\begin{array}{l}\text { Superficial incisional surgical } \\
\text { site infection }\end{array}$ & 364 & 0.55 & 122 & 1.07 & $<0.001$ & 228 & 0.64 & 20 & 1.27 & 0.003 \\
\hline Deep incisional surgical site infection & 92 & 0.14 & 22 & 0.19 & 0.161 & 42 & 0.12 & 6 & 0.38 & 0.004 \\
\hline Organ space surgical site infection & 86 & 0.13 & 22 & 0.19 & 0.093 & 54 & 0.15 & 7 & 0.45 & 0.005 \\
\hline Wound disruption & 128 & 0.19 & 29 & 0.25 & 0.176 & 76 & 0.21 & 4 & 0.25 & 0.731 \\
\hline Pneumonia & 233 & 0.35 & 38 & 0.33 & 0.766 & 104 & 0.29 & 19 & 1.21 & $<0.001$ \\
\hline Unplanned intubation & 108 & 0.16 & 24 & 0.20 & 0.252 & 62 & 0.17 & 8 & 0.51 & 0.003 \\
\hline Pulmonary embolism & 452 & 0.68 & 80 & 0.70 & 0.805 & 230 & 0.65 & 12 & 0.76 & 0.570 \\
\hline On ventilator for more than 48 hours & 50 & 0.08 & 9 & 0.08 & 0.897 & 26 & 0.07 & 6 & 0.38 & $<0.001$ \\
\hline Progressive renal insufficiency & 67 & 0.10 & 34 & 0.30 & $<0.001$ & 42 & 0.12 & 7 & 0.45 & $<0.001$ \\
\hline Acute renal failure & 44 & 0.07 & 14 & 0.12 & 0.041 & 23 & 0.06 & 5 & 0.32 & $<0.001$ \\
\hline Urinary tract infection & 696 & 1.05 & 146 & 1.28 & 0.027 & 380 & 1.07 & 25 & 1.59 & 0.050 \\
\hline Stroke/cerebrovascular accident & 61 & 0.09 & 8 & 0.07 & 0.471 & 35 & 0.10 & 2 & 0.13 & 0.721 \\
\hline Coma for more than 24 hours & 1 & 0.00 & 1 & 0.01 & 0.158 & 1 & 0.00 & 0 & & 0.834 \\
\hline Peripheral nerve injury & 19 & 0.03 & 3 & 0.03 & 0.892 & 6 & 0.02 & 0 & & 0.607 \\
\hline $\begin{array}{l}\text { Cardiac arrest requiring } \\
\text { cardiopulmonary resuscitation }\end{array}$ & 60 & 0.09 & 15 & 0.13 & 0.191 & 43 & 0.12 & 3 & 0.19 & 0.439 \\
\hline Myocardial infarction & 158 & 0.24 & 17 & 0.15 & 0.064 & 76 & 0.21 & 8 & 0.51 & 0.016 \\
\hline $\begin{array}{l}\text { Transfusions/intraoperative/ } \\
\text { postoperative }\end{array}$ & 8008 & 12.06 & 990 & 8.68 & $<0.001$ & 4414 & 12.41 & 279 & 17.77 & $<0.001$ \\
\hline Graft/prosthesis/flap failure & 4 & 0.01 & 0 & & 0.407 & 1 & 0.00 & 0 & & 0.834 \\
\hline $\begin{array}{l}\text { Deep venous thrombosis } \\
\text { requiring therapy }\end{array}$ & 679 & 1.02 & 105 & 0.92 & 0.313 & 379 & 1.07 & 20 & 1.27 & 0.433 \\
\hline Sepsis & 152 & 0.23 & 41 & 0.36 & 0.010 & 91 & 0.26 & 6 & 0.38 & 0.337 \\
\hline Septic shock & 55 & 0.08 & 9 & 0.08 & 0.893 & 28 & 0.08 & 6 & 0.38 & $<0.001$ \\
\hline Mortality & 95 & 0.14 & 16 & 0.14 & 0.942 & 53 & 0.15 & 10 & 0.64 & $<0.001$ \\
\hline Any infection & 1598 & 2.41 & 377 & 3.31 & $<0.001$ & 886 & 2.49 & 79 & 5.03 & $<0.001$ \\
\hline Cardiac pulmonary complication & 713 & 1.07 & 118 & 1.03 & 0.706 & 370 & 1.04 & 27 & 1.72 & 0.010 \\
\hline Any major complication & 956 & 1.44 & 178 & 1.56 & 0.320 & 506 & 1.42 & 37 & 2.36 & 0.003 \\
\hline
\end{tabular}

are considered modifiable risk factors, and some surgeons suggest that they should be corrected before elective TKA $[1,12]$. However, morbid obesity may be less modifiable than malnutrition. Even with intensive and supervised programs involving diet, exercise, lifestyle modification, and/or bariatric surgery, in many cases sustained weight loss has not been achieved in patients with morbid obesity $[13,29,30]$. In addition, some studies have shown that complication rates after TKA in patients whom have undergone bariatric surgery are similar to complication rates for morbidly obese patients who did not undergo bariatric surgery $[13,29]$. We are not aware of any studies in orthopaedics evaluating whether preoperative correction of low serum albumin or nutritional indices results in a decrease in associated complications. However, general surgery studies have shown improvements in serum albumin and other nutritional indices and a corresponding decrease in similar postsurgical complications with 7 to 10 days of either preoperative total enteral nutrition or total parenteral nutrition [4].

Our study has several limitations. First, the study design was retrospective and observational, despite prospective data collection from the NSQIP database. Prospective study may be indicated to further confirm our findings. Second, the hospital information and surgeon identification were removed from NSQIP owing to confidentiality. The quality of various providers might have influenced surgical outcomes, and there was no stratification according to surgeon volume, case-mix complexity, and experience. Third, our study was limited by the number of variables and available data elements in the NSQIP database. Patient albumin levels are not regularly recorded in the NSQIP database, and thus were treated as missing data for certain patients in our analysis. Despite not being a required factor in the NSQIP database, albumin values were present for 37,143 patients $(48 \%)$. While treating missing serum 


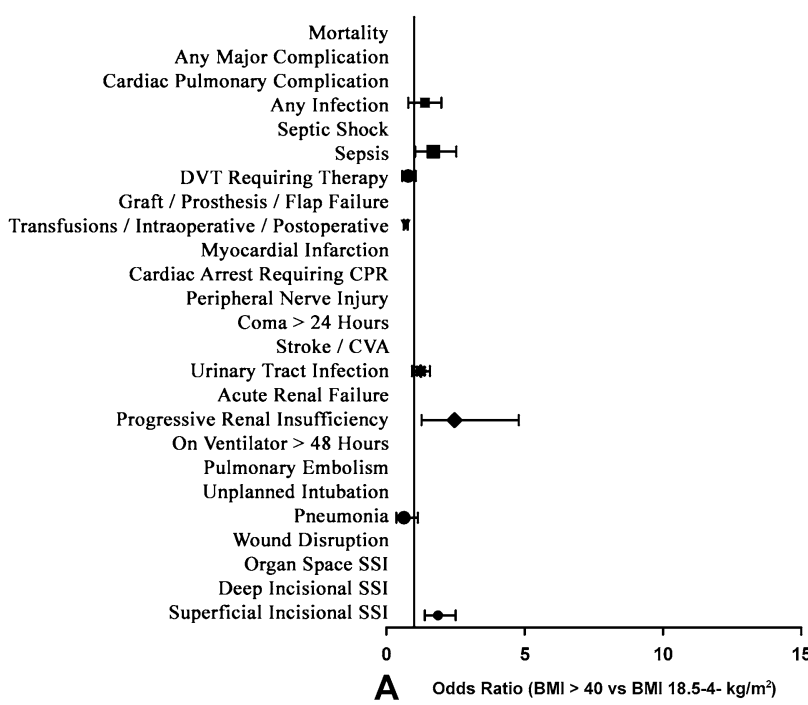

Fig. 2 A-C Odds ratios with 95\% CIs are shown for mortality and selected perioperative complications of (A) morbid obesity versus nonmorbid obesity, (B) serum albumin level less than $3.5 \mathrm{~g} / \mathrm{dL}$ versus serum albumin level of $3.5 \mathrm{~g} / \mathrm{dL}$ or greater, and $(\mathbf{C})$ the interaction of

albumin levels as missing data in the analysis may introduce bias, this results in decreased statistical power only when comparing the association between the low and the normal serum albumin level groups. Therefore, this actually might underestimate the association between low serum albumin and an increase in perioperative complications that was confirmed. However, as BMI data were available for all patients analyzed, the power to detect an increased association between morbid obesity and an increase in perioperative complications was not diminished. Finally, the percentage of patients with low serum albumin in our study $(4.4 \%)$ is similar to percentages reported in another study [12] of total joint arthroplasties. Therefore, we believe this represents a random sampling rather than sampling error [12]. Furthermore, albumin may be critiqued as a marker of nourishment, although as a serum marker, it has been used as a surrogate for nutritional status [21]. Although not all patients with low serum albumin may be malnourished, the data show an association between multiple major perioperative complications and low serum albumin. Finally, the NSQIP collects data for only 30 days after a procedure; thus, results from our study cannot be extrapolated to form conclusions on long-term outcomes.

There have been conflicting studies regarding the association of morbid obesity with increases in short-term and long-term complications after TKA $[3,6,10,13,14,16$, 18, 19, 23-25]. Multivariate analyses to determine specifically whether morbid obesity alone or other comorbid conditions such as malnutrition are the primary

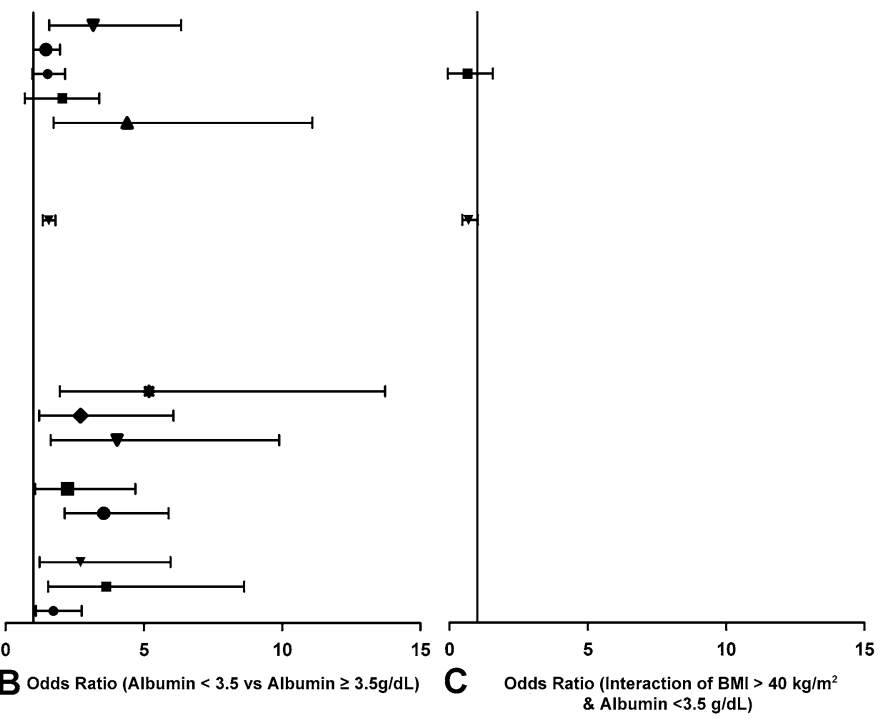

morbid obesity and serum albumin level less than $3.5 \mathrm{~g} / \mathrm{dL}$. $\mathrm{DVT}=$ deep venous thrombosis; $\mathrm{CPR}=$ cardiopulmonary resuscitation; $\quad$ CVA $=$ cerebrovascular accident; $\quad$ SSI $=$ surgical $\quad$ site infection.

factors associated with the increased risk of complications were not performed in some of these studies. Napier et al. [25], in a study of 50 patients who were morbidly obese and 50 who were not morbidly obese followed closely for 1 year, documented no increase in any short-term complications after TKA. However, D'Apuzzo et al. [6], in a much larger study of 180,585 patients using the National Inpatient Sample database, had a higher rate of selected inpatient complications, infection $(0.24 \%$ versus $0.17 \%$, OR, $1.3 ; 95 \% \mathrm{CI}, 1.1-1.7 ; \mathrm{p}=0.001)$, genitourinary-related complications $(0.60 \%$ vs $0.44 \%$, OR, $1.3 ; 95 \%$ CI, $1.1-1.5$; $\mathrm{p}<0.001)$, and postoperative anemia $(16 \%$ vs $15 \%$; OR, $1.0 ; 95 \%$ CI, $1.0-1.1 ; \mathrm{p}<0.001)$. Therefore, despite the differences between the National Inpatient Sample and the NSQIP, they found comparable results to those we documented after multivariate analysis, and similarly showed no increase in numerous serious complications including hematomata, or venous thromboembolic disease and those of the cardiac, peripheral vascular, respiratory, gastrointestinal, or central nervous systems. In contrast to our study, D'Apuzzo et al. [6] did show an increase in mortality. The reason they found an increase in mortality and we did not is unclear, despite the use of large national databases. We suspect that differences in data collection may be responsible since the National Inpatient Sample data elements are limited by the duration of inpatient days, while the NSQIP has the possible advantage of collecting 30 days postoperative information even if patients are discharged from the hospital. In addition, D'Apuzzo et al. [6] studied data from 2005 to 2008 , but we focused on data 
Table 4. Odds ratios of postoperative complications

\begin{tabular}{|c|c|c|c|c|c|c|c|c|c|}
\hline \multirow[t]{2}{*}{ Complication } & \multicolumn{3}{|c|}{$\begin{array}{l}\mathrm{BMI}>40 \mathrm{~kg} / \mathrm{m}^{2} \text { vs } 18.5-40 \\
\mathrm{~kg} / \mathrm{m}^{2}\end{array}$} & \multicolumn{3}{|c|}{ Albumin $<3.5$ vs $\geq 3.5 \mathrm{~g} / \mathrm{dL}$} & \multicolumn{3}{|c|}{$\begin{array}{l}\text { BMI }>40 \mathrm{~kg} / \mathrm{m}^{2} \& \text { Albumin } \\
<3.5 \mathrm{~g} / \mathrm{dL}\end{array}$} \\
\hline & Odds ratio & $\mathrm{p}$ value & $95 \% \mathrm{CI}$ & Odds ratio & $\mathrm{p}$ value & $95 \%$ CI & Odds ratio & $\mathrm{p}$ value & $95 \%$ CI \\
\hline $\begin{array}{l}\text { Superficial incisional surgical } \\
\text { site infection }\end{array}$ & 1.87 & $<0.001$ & $1.39-2.51$ & 1.73 & 0.020 & $1.09-2.75$ & & & \\
\hline Deep incisional surgical site infection & & & & 3.64 & 0.003 & $1.54-8.63$ & & & \\
\hline Organ space surgical site infection & & & & 2.71 & 0.013 & $1.23-5.97$ & & & \\
\hline \multicolumn{10}{|l|}{ Wound disruption } \\
\hline Pneumonia & 0.64 & 0.128 & $0.36-1.14$ & 3.55 & $<0.001$ & $2.14-5.89$ & & & \\
\hline Unplanned intubation & & & & 2.24 & 0.033 & $1.07-4.69$ & & & \\
\hline \multicolumn{10}{|l|}{ Pulmonary embolism } \\
\hline On ventilator for more than 48 hours & & & & 4.03 & 0.002 & $1.64-9.90$ & & & \\
\hline Progressive renal insufficiency & 2.47 & 0.008 & $1.27-4.79$ & 2.71 & 0.015 & $1.21-6.07$ & & & \\
\hline Acute renal failure & & & & 5.19 & 0.001 & $1.96-13.73$ & & & \\
\hline Urinary tract infection & 1.21 & 0.158 & $0.93-1.58$ & & & & & & \\
\hline \multicolumn{10}{|l|}{ Stroke/cerebrovascular accident } \\
\hline \multicolumn{10}{|l|}{ Coma for more than 24 hours } \\
\hline \multicolumn{10}{|l|}{ Peripheral nerve injury } \\
\hline \multicolumn{10}{|l|}{$\begin{array}{l}\text { Cardiac arrest requiring } \\
\text { cardiopulmonary resuscitation }\end{array}$} \\
\hline \multicolumn{10}{|l|}{ Myocardial infarction } \\
\hline $\begin{array}{l}\text { Transfusions/intraoperative/ } \\
\text { postoperative }\end{array}$ & 0.70 & $<0.001$ & $0.63-0.77$ & 1.56 & $<0.001$ & $1.35-1.81$ & 0.69 & 0.064 & $0.47-1.02$ \\
\hline \multicolumn{10}{|l|}{ Graft/prosthesis/flap failure } \\
\hline $\begin{array}{l}\text { Deep venous thrombosis } \\
\text { requiring therapy }\end{array}$ & 0.79 & 0.114 & $0.58-1.06$ & & & & & & \\
\hline Sepsis & 1.70 & 0.034 & $1.04-2.53$ & & & & & & \\
\hline Septic shock & & & & 4.40 & 0.002 & $1.74-11.09$ & & & \\
\hline Mortality & & & & 3.17 & 0.001 & $1.58-6.35$ & & & \\
\hline Any infection & 1.38 & $<0.001$ & $1.16-1.64$ & 2.00 & $<0.001$ & $1.53-2.61$ & 0.58 & 0.072 & $0.32-1.05$ \\
\hline Cardiac pulmonary complication & & & & 1.45 & 0.067 & $0.97-2.15$ & & & \\
\hline Any major complication & & & & 1.41 & 0.050 & $1.00-1.97$ & & & \\
\hline
\end{tabular}

from 2006 to 2013. More complete data and improved perioperative care during the latter period might contribute to some of the differences between these studies. The increase in infection rate was consistent with our findings: however, in our study, the database allowed stratification of infections into superficial infections and more serious deep infections, organ space infections, sepsis, and septic shock. Only superficial infection and sepsis were increased among the morbidly obese cohort in our study. Interpreting our study in the context of prior studies, we believe that morbid obesity is associated with a modest increase in some perioperative complications. Specifically, these complications were superficial infection, sepsis, and progressive renal insufficiency, but there was no independent association with most perioperative complications, including many of the more serious complications such as mortality, thromboembolic disease, cardiac and pulmonary complications, stroke or cerebrovascular accident, peripheral nerve injury, and deep infection. Even with 77,785 patients, we did not have sufficient numbers of patients with BMIs greater than 50 or $60 \mathrm{~kg} / \mathrm{m}^{2}$ to analyze whether super obesity has more of an influence in increasing perioperative complications than morbid obesity. Furthermore, our study is limited to complications within the first 30 days postoperatively, and therefore does not provide insight regarding the presence or absence of an association between morbid obesity and longterm complications after TKA.

Poor nutritional status has been linked to mild and serious adverse outcomes after orthopaedic procedures [17]. Huang et al. [12], in a study of 2161 patients undergoing elective arthroplasties, reported that $8.5 \%$ were malnourished (as defined by low serum albumin level or transferrin values). Jensen et al. [17] suggested that $29 \%$ of patients undergoing elective THAs were malnourished. We found a 
rate of approximately $4.4 \%(1570 / 35,573)$ for low serum albumin in this NSQIP cohort undergoing elective TKAs. Malnutrition is associated with older age, lower immune response, muscle wasting, and poor cardiac function; these comorbidities may further compound the effects of nutritional status and postoperative outcomes [22]. Malnutrition has been linked to persistent wound drainage, failure of wound healing, and surgical site infections after joint arthroplasty $[5,9,11,15]$. Deep periprosthetic infections (acute and chronic) also have been reported at a higher rate in patients with malnutrition [32]. Some studies have concluded that patients with malnutrition had a longer postoperative length of stay [12, 27]. Other complications associated with malnutrition in patients undergoing orthopaedic surgery include postoperative hematoma formation, and renal, cardiovascular, and neurovascular complications [7]. Results of our study are consistent with those of other studies showing an association between low serum albumin and an increase in multiple major perioperative complications [5, 12, 27, 32] To our knowledge, our study is the largest to date evaluating the independent association between low serum albumin and perioperative complications after TKA. We focused specifically on elective primary TKAs and provide a detailed analysis of specific complications which are increased in patients with low serum albumin. In addition, we used multivariate analysis to eliminate potential contributions of morbid obesity and other comorbidities prevalent in patients with morbid obesity. Because of this analysis, we believe there is an association between low serum albumin and multiple major perioperative complications after TKA including mortality, superficial surgical site infection, deep surgical site infection, deep organ space surgical site infection, pneumonia, unplanned intubation required, use of a ventilator more than 48 hours, progressive renal insufficiency, acute renal failure, and septic shock. Patients in the low serum albumin group also were more likely to require blood transfusion and have major complications develop.

Based on these findings, low serum albumin is more important than morbid obesity in increasing the risk of major perioperative complications after TKA. Morbid obesity compared with nonmorbid obesity is associated only with increased rates of progressive renal insufficiency, superficial surgical site infection, and sepsis in patients in the NSQIP database. Restricting access to TKA for patients with morbid obesity who have with advanced OA does not appear indicated, at least regarding concerns related to an increase in major short-term complications. By contrast, it is reasonable to attempt to improve patient nutrition before elective TKA. This is an important finding, as low serum albumin may be a more modifiable risk factor for adverse events after TKA than morbid obesity in patients with immobility and advanced knee OA. Future study is needed to confirm whether preoperative correction of serum albumin level will decrease the risk of complications after TKA.

\section{References}

1. Aggarwal VK, Tischler EH, Lautenbach C, Williams GR Jr, Abboud JA, Altena M, Bradbury TL, Calhoun JH, Dennis DA, Del Gaizo DJ, Font-Vizcarra L, Huotari K, Kates SL, Koo KH, Mabry TM, Moucha CS, Palacio JC, Peel TN, Poolman RN, Robb WJ 3rd, Salvagno R, Seyler T, Skaliczki G, Vasarhelyi EM, Watters WC 3rd. Mitigation and education. J Arthroplasty. 2014;29(2 suppl):19-25.

2. American College of Surgeons; ACS NSQIP ${ }^{\circledR}$. Welcome to the ACS NSQIP. Available at: http://site.acsnsqip.org. Accessed April 13, 2015.

3. Bin Abd Razak HR, Chong HC, Tan AH. Obesity does not imply poor outcomes in Asians after total knee arthroplasty. Clin Orthop Relat Res. 2013;471:1957-1963.

4. Campos AC, Meguid MM. A critical appraisal of the usefulness of perioperative nutritional support. Am J Clin Nutr. 1992;55:117-130.

5. Cross MB, Yi PH, Thomas CF, Garcia J, Della Valle CJ: Evaluation of malnutrition in orthopaedic surgery. J Am Acad Orthop Surg. 2014;22:193-199.

6. D'Apuzzo MR, Novicoff WM, Browne JA. The John Insall Award: Morbid obesity independently impacts complications, mortality, and resource use after TKA. Clin Orthop Relat Res. 2015;473:57-63.

7. Deren ME, Huleatt J, Winkler MF, Rubin LE, Salzler MJ, Behrens SB. Assessment and treatment of malnutrition in orthopaedic surgery. JBJS Reviews. 2014;2:e1. http://dx.doi.org/10.2106/ JBJS.RVW.M.00125.

8. Dewan A, Bertolusso R, Karastinos A, Conditt M, Noble PC, Parsley BS. Implant durability and knee function after total knee arthroplasty in the morbidly obese patient. J Arthroplasty. 2009;24(6 suppl):89-94.

9. Dickhaut SC, DeLee JC, Page CP. Nutritional status: importance in predicting wound-healing after amputation. J Bone Joint Surg Am. 1984;66:71-75.

10. Font-Vizcarra L, Tornero E, Bori G, Bosch J, Mensa J, Soriano A. Relationship between intraoperative cultures during hip arthroplasty, obesity, and the risk of early prosthetic joint infection: a prospective study of 428 patients. Int J Artif Organs. 2011;34:870-875.

11. Greene KA, Wilde AH, Stulberg BN. Preoperative nutritional status of total joint patients: relationship to postoperative wound complications. J Arthroplasty. 1991;6:321-325.

12. Huang R, Greenky M, Kerr GJ, Austin MS, Parvizi J. The effect of malnutrition on patients undergoing elective joint arthroplasty. J Arthroplasty. 2013;28(8 suppl):21-24.

13. Inacio MC, Paxton EW, Fisher D, Li RA, Barber TC, Singh JA. Bariatric surgery prior to total joint arthroplasty may not provide dramatic improvements in post-arthroplasty surgical outcomes. $J$ Arthroplasty. 2014;29:1359-1364.

14. Issa K, Pivec R, Kapadia BH, Shah, T, Harwin, SF, Delanois, RE, Mont, MA. Does obesity affect the outcomes of primary total knee arthroplasty? J Knee Surg. 2013;26:89-94.

15. Jaberi FM, Parvizi J, Haytmanek CT, Joshi A, Purtill J. Procrastination of wound drainage and malnutrition affect the outcome of joint arthroplasty. Clin Orthop Relat Res. 2008;466:1368-1371.

16. Jarvenpaa J, Kettunen J, Kroger H, Miettinen H. Obesity may impair the early outcome of total knee arthroplasty. Scand J Surg. 2010;99:45-49. 
17. Jensen JE, Jensen TG, Smith TK, Johnston DA, Dudrick SJ. Nutrition in orthopaedic surgery. J Bone Joint Surg Am. 1982;64: $1263-1272$.

18. Kerkhoffs GM, Servien E, Dunn W, Dahm D, Bramer JA, Haverkamp D. The influence of obesity on the complication rate and outcome of total knee arthroplasty: a meta-analysis and systematic literature review. J Bone Joint Surg Am. 2012;94: 1839-1844.

19. Kremers HM, Visscher SL, Kremers WK, Naessens JM, Lewallen DG. The effect of obesity on direct medical costs in total knee arthroplasty. J Bone Joint Surg Am. 2014;96:718-724.

20. Krushell RJ, Fingeroth RJ. Primary total knee arthroplasty in morbidly obese patients: a 5 - to 14 -year follow-up study. $J$ Arthroplasty. 2007;22(6 suppl 2):77-80.

21. Kudsk KA, Tolley EA, DeWitt RC, Janu PG, Blackwell AP, Yeary S, King BK. Preoperative albumin and surgical site identify surgical risk for major postoperative complications. JPEN $J$ Parenter Enteral Nutr. 2003;27:1-9.

22. Lesourd B, Mazari L. Nutrition and immunity in the elderly. Proc Nutr Soc. 1999;58:685-695.

23. Memtsoudis SG, Bombardieri AM, Ma Y, Walz JM, Chiu YL, Mazumdar M. Mortality of patients with respiratory insufficiency and adult respiratory distress syndrome after surgery: the obesity paradox. J Intensive Care Med. 2012;27:306-311.

24. Memtsoudis SG, Gonzalez Della Valle A, Besculides MC, Gaber L, Sculco TP. In-hospital complications and mortality of unilateral, bilateral, and revision TKA: based on an estimate of 4,159,661 discharges. Clin Orthop Relat Res. 2008;466:2617-2627.

25. Napier RJ, O'Brien S, Bennett D, Doran E, Sykes A, Murray J, Beverland DE. Intra-operative and short term outcome of total knee arthroplasty in morbidly obese patients. Knee. 2014; 21 : 784-788.

26. Naziri Q, Issa K, Malkani AL, Bonutti PM, Harwin SF, Mont MA. Bariatric orthopaedics: total knee arthroplasty in superobese patients (BMI $>50 \mathrm{~kg} / \mathrm{m} 2$ ). Survivorship and complications. Clin Orthop Relat Res. 2013;471:3523-3530.

27. Nicholson JA, Dowrick AS, Liew SM. Nutritional status and short-term outcome of hip arthroplasty. J Orthop Surg (Hong Kong). 2012;20:331-335.

28. Rajgopal V, Bourne RB, Chesworth BM, MacDonald SJ, McCalden RW, Rorabeck CH. The impact of morbid obesity on patient outcomes after total knee arthroplasty. J Arthroplasty. 2008;23:795-800.

29. Severson EP, Singh JA, Browne JA, Trousdale RT, Sarr MG, Lewallen DG. Total knee arthroplasty in morbidly obese patients treated with bariatric surgery: a comparative study. J Arthroplasty. 2012;27:1696-1700.

30. Unick JL, Beavers D, Bond DS, Clark JM, Jakicic JM, Kitabchi AE, Knowler WC, Wadden TA, Wagenknecht LE, Wing RR; Look AHEAD Research Group. The long-term effectiveness of a lifestyle intervention in severely obese individuals. Am J Med. 2013;126:236-242.

31. Workgroup of the American Association of Hip and Knee Surgeons Evidence Based Committee. Obesity and total joint arthroplasty: a literature based review. J Arthroplasty. 2013;28: 714-721.

32. Yi PH, Frank RM, Vann E, Sonn KA, Moric M, Della Valle CJ. Is potential malnutrition associated with septic failure and acute infection after revision total joint arthroplasty? Clin Orthop Relat Res. 2015;473:175-182. 